
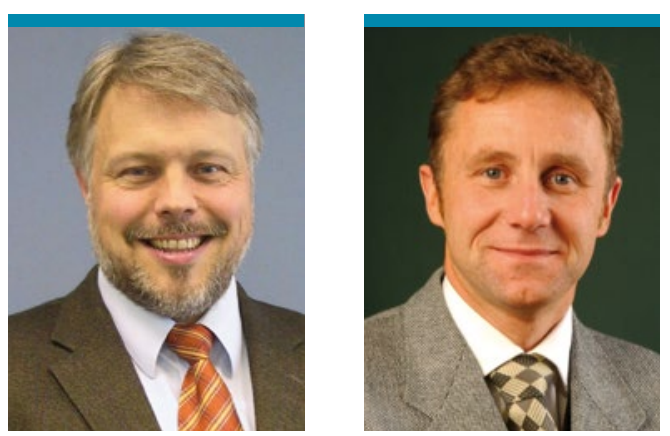

"Höhepunkte des Mainzer Allergie-Workshops werden auch dieses Jahr wieder der Junior-Members-Workshop und die Vergabe des Mainzer-Abstract-Preises der DGAKI sein."

Prof. Dr. Joachim Saloga, Tagungsleiter

Prof. Dr. Stephan Grabbe, Tagungspräsident

\title{
28. Mainzer Allergie-Workshop
}

$\mathrm{F}$ ür den 28. Mainzer Allergie-Workshop, die wissenschaftliche Frühjahrstagung der Deutschen Gesellschaft für Allergologie und klinische Immunologie (DGAKI), die am 17. und 18. März stattfindet, sind auch in diesem Jahr wieder zahlreiche interessante Abstracts eingereicht worden, aus denen wir ein wissenschaftlich sehr hochrangiges Programm zusammenstellen konnten. Dieses reicht von den immunologischen Grundlagen und Allergenen über die Nahrungsmittelallergie hin zu den allergischen Krankheitsbildern an den Atemwegen und an der Haut sowie allgemeiner Allergiediagnostik und -therapie. Auch in diesem Jahr werden die einzelnen Themensitzungen durch kurze Impulsreferate der Vorsitzenden eingeleitet. Das vielgestaltige Programm sollte daher Grundlagenforscher ebenso ansprechen wie pathophysiologisch und molekular orientierte klinische Spezialisten. Der Workshop wird

- wie immer - in Kooperation mit der Österreichischen und Schweizerischen Gesellschaft für Allergologie und Klinische Immunologie (ÖGAI und SGAI) veranstaltet. Das Layout des Programmheftes orientiert sich am einheitlichen Kongress- und Fortbildungs-Layout der DGAKI, wobei alle Veranstaltungen unter dem Oberbegriff der Allergieakademie der

\section{Wissenschaftliches Programm und Inhaltsverzeichnis}

\begin{tabular}{|lcc|}
\hline Vorträge & Nummer & Seite \\
\hline Immunologie & V01-V06 & 38 \\
\hline Allergene & V07-V13 & 40 \\
Nahrungsmittel/Gastrointestinaltrakt & V14-V22 & 43 \\
Atemwege & V23-V31 & 47 \\
Haut & V32-V37 & 50 \\
Diagnostik und Therapie & V38-V44 & 52 \\
\hline Poster & P01-P06 & 55 \\
\hline Verzeichnis der Autoren & & 37 \\
\hline Alle Abstracts erscheinen parallel im Allergo Journal International [Allergo J Int 2016;25:18-37].
\end{tabular}

DGAKI zusammengeführt werden. Die Frühjahrstagung stellt dabei die allgemeine grundlagenwissenschaftliche Tagung mit Workshop-Charakter dar, bei der insbesondere auch junge Forscher ihre neuesten Ergebnisse vortragen.

Wie in den letzten Jahren, findet auch in diesem Jahr der Workshop wieder Donnerstag und Freitag statt, weil dies von den meisten Teilnehmern bevorzugt wird. Da sich in den letzten Jahren der geführte Posterrundgang sehr bewährt hat, wollen wir diese Tradition ebenfalls weiter fortsetzen. Parallel zum Programm haben die einzelnen Sektionen und Arbeitsgruppen der DGAKI Gelegenheit zum Tagen. Höhepunkte werden auch dieses Jahr wieder der Junior-Members-Workshop und die nachfolgende Vergabe des Mainzer-Abstract-Preises der DGAKI darstellen, der freundlicher Weise von der Firma ALK-Abelló für die Prämierung der besten eingereichten Abstracts gestiftet wurde.

Durch die finanziellen Beiträge der ausstellenden Firmen, deren Präsentationen wir entsprechend $\mathrm{zu}$ beachten bitten, ist es uns auch weiterhin möglich, den Tagungsbeitrag mit $25 €$ pro Person sehr niedrig zu halten und dennoch einen Gesellschaftsabend am Donnerstagabend (diesmal im Hilton-Hotel am Rhein) zu veranstalten. Dort sollen alle Teilnehmer die Möglichkeit haben, ihre wissenschaftlichen Gespräche fortzusetzen, Kooperationen anzubahnen und sich auch persönlich näher kennenzulernen.

Schließlich wurde auch wieder die Zertifizierung des Workshops bei der hiesigen Ärztekammer beantragt.

Wir freuen uns auf ein baldiges Zusammentreffen auf einem sicherlich wieder sehr stimulierenden Workshop in Mainz, zu dem wir Sie herzlich einladen.
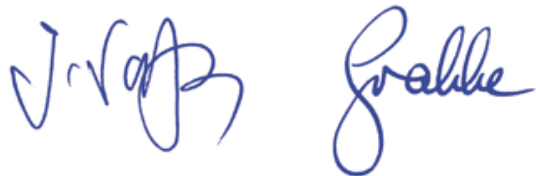